\title{
On vibrations in thermoelasticity without energy dissipation for micropolar bodies
}

\author{
Marin Marin ${ }^{1 *}$ and Dumitru Baleanu $u^{2,3}$
}

\section{${ }^{*}$ Correspondence:}

m.marin@unitbv.ro

'Department of Mathematics and

Computer Science, Transilvania

University of Brasov, Brasov, 500118 , Romania

Full list of author information is

available at the end of the article

\begin{abstract}
We consider a micropolar thermoelastic body occupying a prismatic cylinder that is free of loads on lateral surface (no body force, no body couple, and no heat supply). On the base of the cylinder are prescribed a time-dependent displacement, a microrotation, and a thermal displacement, which are harmonic in time, and collaborate to induce the motion of the considered body. With the help of a measure associated with the corresponding steady-state vibration and by assuming that the exciting frequency is lower than a certain critical frequency, we will obtain a spatial decay estimate.
\end{abstract}

Keywords: thermoelasticity; micropolar; energy dissipation; vibration

\section{Introduction}

The theory of thermoelastic material behavior without energy dissipation possesses the following properties: the heat flow, in contrast to that in classical thermoelasticity characterized by the Fourier law, does not involve energy dissipation; a constitutive equation for an entropy flux vector is determined by the same potential function as also determines the stress, and it permits the transmission of heat as thermal waves at finite speed.

It is well known that in a micropolar continuum the deformation is described not only by the displacement vector but also by an independent rotation vector. This rotation vector specifies the orientation of a triad of director vectors attached to each material particle. A material point can experience a microrotation without undergoing a macrodisplacement. An infinitesimal surface element transmits a force and a couple vector, which give rise to nonsymmetric stress and couple-stress tensors. The former is related to a nonsymmetric strain tensor and the latter to a nonsymmetric curvature tensor, defined as the gradient of the rotation vector.

It is believed that this type of the continuum mechanics was originally introduced by Voigt since 1887 and the brothers Cosserat since 1909. There is a simplified variant of the theory of micropolar bodies, the so-called couple-stress theory, and in this theory the rotation vector is not independent of the displacement vector, but related to it in the same way as in classical continuum mechanics. The motivation for the extension of the classical to micropolar and couple-stress theory was that the classical theory was not able to predict the size effect experimentally observed in problems which had a geometric length scale comparable to the material's microstructural length, such as the grain size in a polycrystalline or granular aggregate. For example, the apparent strength of some materials

(c) 2016 Marin and Baleanu. This article is distributed under the terms of the Creative Commons Attribution 4.0 International License (http://creativecommons.org/licenses/by/4.0/), which permits unrestricted use, distribution, and reproduction in any medium, provided you give appropriate credit to the original author(s) and the source, provide a link to the Creative Commons license, and indicate if changes were made. 
with stress concentrators such as holes and notches is higher for smaller grain size; for a given volume fraction of dispersed hard particles, the strengthening of metals is greater for smaller particles; the bending and torsional strengths are higher for very thin beams and wires.

There are several hyperbolic theories for describing the heat conduction which are also called theories of second sound. In these theories the flow of heat is modeled with finite propagation speed, in contrast to the classical model based on the Fourier law, leading to infinite propagation speed of heat signals. A review of these theories is presented in the paper [1] by Chandrasekharaiah.

First results in the thermoelastic theory without energy dissipation were obtained by Green and Naghdi [2]. This thermomechanical theory of deformable media introduces the so-called thermal displacement related to the common temperature. and uses a general entropy balance as postulated in Green and Naghdi [3]. By the procedure of Green and Naghdi, the reduced energy equation is regarded as an identity for all thermodynamical processes and places some restrictions on the functional forms of the dependent constitutive variables. The theory is illustrated in detail in the context of the flow of heat in a rigid solid, with particular reference to the propagation of s thermal waves at finite speed. The linear theory of thermoelasticity without energy dissipation for homogeneous and isotropic materials was employed by Nappa [4] to obtain spatial energy bounds and decay estimates for the transient solutions in connection with the problem in which a thermoelastic body is deformed subject to boundary and initial data and body supplies having a compact support, provided positive definiteness assumptions are made upon the constitutive coefficients. Also, in the linear theory of thermoelasticity without energy dissipation Chandrasekharaiah [5] proves the uniqueness of the solutions, Iesan [6] establishes continuous dependence results, while Quintanilla [7] studies the question of existence. In [8-10] we find some results regarding vibrations for magneto-thermoelastic bodies. Other results regarding thermoelasticity of dipolar bodies and of microstretch bodies are presented in [11-16]. Some concrete and practical issues related to porous media can be found in [17] and [18].

Our present study is dedicated to the spatial behavior of the harmonic in time vibrations within the model of the linear thermoelasticity theory without dissipation energy for micropolar bodies. We prove a priori estimates for the amplitude of a harmonic vibration by means of some auxiliary identities. It provides some estimates describing how the amplitude evolves with respect to the distance to the excited base, provided the frequency of vibration is greater than a certain critical value.

The spatial behavior of the harmonic in time vibrations has been studied by Chirita [19] in the theory of classical linear thermoelasticity. Here the author uses a technique developed by Flavin and Knops [20] in the low frequency range. Some differential inequalities are established for the appropriate selected measures which after integration provide exponential estimates for the spatial evolution of the amplitude of vibration, provided the positive definiteness of the constitutive coefficients is assumed.

In [21] Ciarletta proposed a theory of micropolar thermoelasticity, which, because it is a theory without energy dissipation, allows propagation of thermal waves at a finite speed.

The plan of the paper is the following. We first write down the mixed initial boundary value problem within context of micropolar bodies in thermoelasticity without energy dissipation. Then we prove some differential relations for certain cross-sectional integrals. 
Based on these relations we obtain estimates describing how the amplitude evolves with respect to the distance to the excited base. This requires the frequency of vibrations to be greater than a certain critical value.

\section{Basic equations}

Let $B$ be an open set domain of a three-dimensional Euclidian space occupied by the reference configuration of a homogeneous micropolar body. We assume that $B$ is regular and a finite region with boundary $\partial B$ and we denote the closure of $B$ by $\bar{B}$. We use a fixed system of rectangular Cartesian axes and adopt Cartesian tensor notation. Points in $B$ are denoted by $x_{j}$ and $t \in[0, \infty)$ is the temporal variable. Also, the spatial argument and the time argument of a function will be omitted when there is no likelihood of confusion. A superposed dot denotes the differentiation with respect to time $t$, and a subscript preceded by a comma denotes the differentiation with respect to the corresponding spatial variable.

The governing equations of the theory of anisotropic and homogeneous micropolar thermoelasticity without energy dissipation, as we can find in [21], consist of the equations of motion

$$
\begin{aligned}
& t_{i j, j}+\varrho F_{i}=\varrho \ddot{u}_{i}, \\
& m_{i j, j}+\varepsilon_{i j k} t_{j k}+\varrho M_{i}=I_{i j} \ddot{\varphi}_{j} ;
\end{aligned}
$$

and the equation of energy

$$
\varrho \dot{\eta}=\frac{\varrho}{\theta_{0}} r-q_{i, i}
$$

Equations (1) and (2) are defined for $(x, t) \in B \times(0, \infty)$.

When the reference solid has a center of symmetry at each point but is otherwise nonisotropic, the constitutive equations, defined for $(x, t) \in \bar{B} \times[0, \infty)$, are

$$
\begin{aligned}
& t_{i j}=A_{i j m n} \varepsilon_{m n}+B_{i j m n} \gamma_{m n}-D_{i j} \theta, \\
& m_{i j}=B_{m n i j} \varepsilon_{m n}+C_{i j m n} \gamma_{m n}-E_{i j} \theta, \\
& \varrho \eta=D_{i j} \varepsilon_{i j}+E_{i j} \gamma_{i j}+\frac{c}{\theta_{0}} \theta, \\
& q_{i}=-\frac{1}{\theta_{0}} K_{i j} \beta_{j} .
\end{aligned}
$$

The deformation tensors $\varepsilon_{i j}$ and $\gamma_{i j}$ used in equations (3) are defined, in $\bar{B} \times[0, \infty)$, by means of the geometric equations

$$
\varepsilon_{i j}=u_{j, i}+\varepsilon_{j i k} \varphi_{k}, \quad \gamma_{i j}=\varphi_{j, i}
$$

The system of equations is complete if we add the law of heat flow

$$
\dot{\beta}_{i}=\theta_{, i}
$$

for all $(x, t) \in \bar{B} \times[0, \infty)$. 
In the equations above we have used the following notation: $u_{i}$ for the components of displacement vector, $\varphi_{i}$ for the components of microrotatia vector, $t_{i j}$ for the components of stress tensor, $m_{i j}$ for the components of couple-stress tensor, $q_{i}$ for the components of the heat conduction vector, $\eta$ for the specific entropy per unit mass, $\varrho$ for the constant reference density mass, $\theta_{0}$ for the constant reference temperature, $I_{i j}$ for the components of inertia, $\beta_{i}$ for the components of the thermal displacement gradient vector, $F_{i}$ for the components of the external body force vector, $M_{i}$ for the components of the external body couple vector, $r$ for the external rate of supply of heat per unit mass, and $\varepsilon_{i j k}$ is the alternating symbol.

The coefficients from (3), that is, $A_{i j m n}, B_{i j m n}, C_{i j m n}, D_{i j}, E_{i j}$, c, and $K_{i j}$ are constant constitutive coefficients subject to the following symmetry conditions:

$$
A_{i j m n}=A_{m n i j}, \quad C_{i j m n}=C_{m n i j}, \quad I_{i j}=I_{j i}, \quad K_{i j}=K_{j i} .
$$

The free energy $\Psi$, used to obtain the constitutive equations, is given by

$$
\begin{aligned}
\varrho \Psi= & \frac{1}{2} A_{i j m n} \varepsilon_{i j} \varepsilon_{m n}+B_{i j m n} \varepsilon_{i j} \gamma_{m n}+\frac{1}{2} C_{i j m n} \gamma_{i j} \gamma_{m n} \\
& -D_{i j} \varepsilon_{i j} \theta-E_{i j} \gamma_{i j} \theta-\frac{c}{2 \theta_{0}} \theta^{2}+\frac{c}{2 \theta_{0}} K_{i j} \tau_{, i} \tau_{, j} .
\end{aligned}
$$

Here we denote by $\tau$ the thermal displacement related to the temperature variation. The relationship between $\tau$ and $\theta$ is given by

$$
\dot{\tau}=\theta \text {. }
$$

Introducing the constitutive equations (3) and the geometric equations (4) in the equations of motion (1) and the equation of energy (2), we obtain a system of equations in terms of displacements $u_{i}$, microrotations $\varphi_{i}$, and thermal displacements $\tau$ as

$$
\begin{aligned}
& {\left[A_{i j m n}\left(u_{n, m}+\varepsilon_{m n k} \varphi_{k}\right)+B_{i j m n} \varphi_{n, m}-D_{i j} \dot{\tau}\right]_{, j}+\varrho F_{i}=\varrho \ddot{u} i,} \\
& {\left[B_{m n i j}\left(u_{n, m}+\varepsilon_{m n k} \varphi_{k}\right)+C_{i j m n} \varphi_{n, m}-E_{i j} \dot{\tau}\right]_{, j}} \\
& \quad+\varepsilon_{i j k}\left[A_{j k m n}\left(u_{n, m}+\varepsilon_{m n k} \varphi_{k}\right)+B_{j k m n} \varphi_{n, m}-D_{j k} \dot{\tau}\right]+\varrho M_{i}=I_{i j} \ddot{\varphi}_{j}, \\
& \frac{1}{\theta_{0}}\left(K_{i j} \tau_{, j}\right)_{, i}-D_{i j}\left(\dot{u}_{j, i}+\varepsilon_{j i k} \dot{\varphi}_{k}\right)-E_{i j} \dot{\varphi}_{j, i}+\frac{\varrho}{\theta_{0}} r=\frac{c}{\theta_{0}} \ddot{\tau},
\end{aligned}
$$

for any $(x, t) \in B \times(0, \infty)$.

\section{Preliminary results}

Consider a cross-section $D$ of a prismatic cylinder and the boundary of the section, $\partial D$, assumed to be piecewise continuously differentiable. We choose the system of Cartesian rectangular axis so that its origin is in the center of the cylinder base and the positive $x_{3}$ axis is directed along the cylinder. If we denote by $L$ the length of the cylinder, then the lateral boundary of the cylinder is $\mathcal{S}=\partial D \times[0, L]$. The contents of the prismatic cylinder is a micropolar thermoelastic body which is homogeneous and anisotropic. 
The cylinder is free of load on the lateral boundary surface, that is, we have a zero body force, couple force and heat supply and zero displacement, microrotations, and thermal displacements. But over the base of cylinder are specified the displacements, microrotations, and thermal displacement, all of which are assumed to be harmonic in time. Therefore, besides the system of equations (9) we can adjoin the following lateral boundary conditions:

$$
u_{i}(x, t)=0, \quad \varphi_{i}(x, t)=0, \quad \tau(x, t)=0, \quad(x, t) \in \mathcal{S} \times(0, \infty),
$$

and the base boundary conditions

$$
\begin{aligned}
& u_{i}\left(x_{1}, x_{2}, 0, t\right)=\tilde{u}_{i}\left(x_{1}, x_{2}\right) e^{i \omega t}, \quad \varphi_{i}\left(x_{1}, x_{2}, 0, t\right)=\tilde{\varphi}_{i}\left(x_{1}, x_{2}\right) e^{i \omega t}, \\
& \tau\left(x_{1}, x_{2}, 0, t\right)=\tilde{t}\left(x_{1}, x_{2}\right) e^{i \omega t}, \quad\left(x_{1}, x_{2}\right) \in D(0), t>0,
\end{aligned}
$$

where $\tilde{u}_{i}\left(x_{1}, x_{2}\right), \tilde{\varphi}_{i}\left(x_{1}, x_{2}\right)$, and $\tilde{t}\left(x_{1}, x_{2}\right)$ are prescribed smooth functions, $\iota$ is the complex unit, and $\omega$ is a prescribed positive constant.

Loads from (11) induce inside the cylinder some vibrations harmonic in time, having the form

$$
\begin{aligned}
& u_{i}\left(x_{1}, x_{2}, x_{3}, t\right)=U_{i}\left(x_{1}, x_{2}, x_{3}\right) e^{i \omega t}, \quad \varphi_{i}\left(x_{1}, x_{2}, x_{3}, t\right)=\Phi_{i}\left(x_{1}, x_{2}, x_{3}\right) e^{i \omega t}, \\
& \tau\left(x_{1}, x_{2}, x_{3}, t\right)=T\left(x_{1}, x_{2}, x_{3}\right) e^{i \omega t}, \quad\left(x_{1}, x_{2}, x_{3}, t\right) \in B \times(0, \infty) .
\end{aligned}
$$

The amplitude $\left(U_{i}, \Phi_{i}, T\right)$ of the vibrations satisfies the following system of differential equations:

$$
\begin{aligned}
& {\left[A_{i j m n}\left(U_{n, m}+\varepsilon_{m n k} \Phi_{k}\right)+B_{i j m n} \Phi_{n, m}-\iota \omega D_{i j} T\right]_{, j}+\varrho \omega^{2} U_{i}=0,} \\
& {\left[B_{m n i j}\left(U_{n, m}+\varepsilon_{m n k} \Phi_{k}\right)+C_{i j m n} \Phi_{n, m}-\iota \omega E_{i j} T\right]_{, j}} \\
& \quad+\varepsilon_{i j k}\left[A_{j k m n}\left(U_{n, m}+\varepsilon_{m n k} \Phi_{k}\right)+B_{j k m n} \Phi_{n, m}-\iota \omega D_{j k} T\right]+I_{i j} \omega^{2} \Phi_{j}=0, \\
& \left(\frac{1}{\theta_{0}} K_{i j} T_{, j}\right)_{, i}-\iota \omega D_{i j}\left(U_{j, i}+\varepsilon_{j i k} \Phi_{k}\right)-\iota \omega E_{i j} \Phi_{j, i}+\frac{c}{\theta_{0}} \omega^{2} T=0 .
\end{aligned}
$$

The lateral boundary conditions get the form

$$
U_{i}(x)=0, \quad \Phi_{i}(x)=0, \quad T(x)=0, \quad x \in \mathcal{S},
$$

and the base boundary conditions become

$$
\begin{aligned}
& U_{i}\left(x_{1}, x_{2}, 0\right)=\tilde{U}_{i}\left(x_{1}, x_{2}\right), \quad \Phi_{i}\left(x_{1}, x_{2}, 0\right)=\tilde{\Phi}_{i}\left(x_{1}, x_{2}\right), \\
& T\left(x_{1}, x_{2}, 0\right)=\tilde{T}\left(x_{1}, x_{2}\right), \quad\left(x_{1}, x_{2}\right) \in D(0) .
\end{aligned}
$$

In the case of a finite cylinder we will have to prescribe a boundary condition on the superior base of the cylinder, $D(L)$. For a forced oscillation the spatial behavior of the amplitude was studied by Chirita [19] and Ciarletta [21], in the case of a rhombic thermoelastic material, provided the exciting frequency is less than a certain critical frequency. The main goal 
of our study is to estimate how the amplitude evolves with respect to the axial distance to the excited and.

In the following we want to prove some estimates on a solution of the system of equations (13), with the lateral boundary conditions (14) and the base boundary conditions (15). We will use the notation $\mathcal{U}_{j, i}=U_{j, i}+\varepsilon_{j i k} \Phi_{k}$.

In the following theorem we will state and prove four auxiliary identities on which will be based the main result.

Theorem 1 Let $\left(U_{i}, \Phi_{i}, T\right)$ be a solution of the boundary value problem consisting of equations (13)-(15). Then the following equalities are satisfied:

$$
\begin{aligned}
& 2 \int_{D\left(x_{3}\right)}\left\{A_{i j m n} \mathcal{U}_{j, i} \overline{\mathcal{U}}_{n, m}+C_{i j m n} \Phi_{n, m} \bar{\Phi}_{j, i}\right. \\
& \left.+B_{i j m n}\left[\mathcal{U}_{j, i} \bar{\Phi}_{n, m}+\overline{\mathcal{U}}_{j, i} \Phi_{n, m}\right]-\varrho \omega^{2} U_{i} \bar{U}_{i}-I_{i j} \omega^{2} \Phi_{i} \bar{\Phi}_{j}\right\} d A \\
& +\int_{D\left(x_{3}\right)}\left\{\iota \omega D_{i j}\left(\bar{T} \mathcal{U}_{j, i}-T \overline{\mathcal{U}}_{j, i}\right)+\iota \omega E_{i j}\left(\bar{T} \Phi_{i, j}-T \bar{\Phi}_{i, j}\right)\right\} d A \\
& =\frac{d}{d x_{3}} \int_{D\left(x_{3}\right)}\left\{\left[A_{3 j m n} \mathcal{U}_{n, m}+B_{3 j m n} \Phi_{n, m}-\iota \omega D_{3 j} T\right] \bar{U}_{j}\right\} d A \\
& +\frac{d}{d x_{3}} \int_{D\left(x_{3}\right)}\left\{\left[A_{3 j m n} \overline{\mathcal{U}}_{n, m}+B_{3 j m n} \bar{\Phi}_{n, m}+\iota \omega D_{3 j} \bar{T}\right] U_{j}\right\} d A \\
& +\frac{d}{d x_{3}} \int_{D\left(x_{3}\right)}\left\{\left[B_{3 j m n} \mathcal{U}_{n, m}+C_{3 j m n} \Phi_{n, m}-\iota \omega E_{3 j} T\right] \bar{\Phi}_{j}\right\} d A \\
& +\frac{d}{d x_{3}} \int_{D\left(x_{3}\right)}\left\{\left[B_{3 j m n} \overline{\mathcal{U}}_{n, m}+C_{3 j m n} \bar{\Phi}_{n, m}+\iota \omega E_{3 j} \bar{T}\right] \Phi_{j}\right\} d A, \\
& \int_{D\left(x_{3}\right)}\left[\iota \omega D_{i j}\left(\bar{T} \mathcal{U}_{j, i}+T \overline{\mathcal{U}}_{j, i}\right)+\iota \omega E_{i j}\left(\bar{T} \Phi_{i, j}+T \bar{\Phi}_{i, j}\right)\right] d A \\
& =\frac{d}{d x_{3}} \int_{D\left(x_{3}\right)}\left\{\left[A_{3 j m n} \overline{\mathcal{U}}_{n, m}+B_{3 j m n} \bar{\Phi}_{n, m}+\iota \omega D_{3 j} \bar{T}\right] U_{j}\right\} d A \\
& -\frac{d}{d x_{3}} \int_{D\left(x_{3}\right)}\left\{\left[A_{3 j m n} \mathcal{U}_{n, m}+B_{3 j m n} \Phi_{n, m}-\iota \omega D_{3 j} T\right] \bar{U}_{j}\right\} d A \\
& +\frac{d}{d x_{3}} \int_{D\left(x_{3}\right)}\left\{\left[B_{3 j m n} \overline{\mathcal{U}}_{n, m}+C_{3 j m n} \bar{\Phi}_{n, m}+\iota \omega E_{3 j} \bar{T}\right] \Phi_{j}\right\} d A \\
& -\frac{d}{d x_{3}} \int_{D\left(x_{3}\right)}\left\{\left[B_{3 j m n} \mathcal{U}_{n, m}+C_{3 j m n} \Phi_{n, m}-\iota \omega E_{3 j} T\right] \bar{\Phi}_{j}\right\} d A, \\
& 2 \int_{D\left(x_{3}\right)} \frac{1}{\theta_{0}}\left(K_{i j} T_{, i} \bar{T}_{, j}-c \omega^{2} T \bar{T}\right) d A+\int_{D\left(x_{3}\right)} \iota \omega E_{i j}\left(\Phi_{j, i} \bar{T}-\bar{\Phi}_{j, i} T\right) d A \\
& +\int_{D\left(x_{3}\right)} \iota \omega D_{i j}\left(\mathcal{U}_{j, i} \bar{T}-\overline{\mathcal{U}}_{j, i} T\right) d A=\frac{d}{d x_{3}} \int_{D\left(x_{3}\right)} \frac{1}{\theta_{0}} K_{33}\left(\bar{T} T_{, 3}+T \bar{T}_{, 3}\right) d A, \\
& \int_{D\left(x_{3}\right)} \iota \omega D_{i j}\left(\mathcal{U}_{j, i} \bar{T}+\mathcal{U}_{j, i} T\right) d A \\
& +\int_{D\left(x_{3}\right)} \iota \omega E_{i j}\left(\bar{\Phi}_{j, i} T+\Phi_{j, i} \bar{T}\right) d A=\frac{d}{d x_{3}} \int_{D\left(x_{3}\right)} \frac{1}{\theta_{0}} K_{3 j}\left(\bar{T} T_{, j}-T \bar{T}_{, j}\right) d A,
\end{aligned}
$$

where $\bar{z}$ is the notation for the complex conjugate of $z$. 
Proof Considering equations $(13)_{1}$ and $(13)_{2}$ we can prove the following equality:

$$
\begin{aligned}
\{ & {\left.\left[A_{i j m n} \mathcal{U}_{n, m}+B_{i j m n} \Phi_{n, m}-\iota \omega D_{i j} T\right]_{, j}+\varrho \omega^{2} U_{i}\right\} \bar{U}_{i} } \\
& +\left\{\left[A_{i j m n} \overline{\mathcal{U}}_{n, m}+B_{i j m n} \bar{\Phi}_{n, m}+\iota \omega D_{i j} \bar{T}\right]_{, j}+\varrho \omega^{2} \bar{U}_{i}\right\} U_{i} \\
& +\left[B_{m n i j} \mathcal{U}_{n, m}+C_{i j m n} \Phi_{n, m}-\iota \omega E_{i j} T\right]_{, j} \bar{\Phi}_{i} \\
& +\varepsilon_{i j k}\left[A_{j k m n} \mathcal{U}_{n, m}+B_{j k m n} \Phi_{n, m}-\iota \omega D_{j k} T\right] \bar{\Phi}_{i}+I_{i j} \omega^{2} \Phi_{i} \bar{\Phi}_{j} \\
& +\left[B_{m n i j} \overline{\mathcal{U}}_{n, m}+C_{i j m n} \bar{\Phi}_{n, m}+\iota \omega E_{i j} \bar{T}\right]_{, j} \Phi_{i} \\
& +\varepsilon_{i j k}\left[A_{j k m n} \overline{\mathcal{U}}_{n, m}+B_{j k m n} \bar{\Phi}_{n, m}+\iota \omega D_{j k} \bar{T}\right] \Phi_{i}+I_{i j} \omega^{2} \Phi_{i} \bar{\Phi}_{j}=0 .
\end{aligned}
$$

With some calculations, equality (20) can be written in the form

$$
\begin{aligned}
2\left\{A_{i j m n} \mathcal{U}_{j, i} \overline{\mathcal{U}}_{n, m}+C_{i j m n} \Phi_{n, m} \bar{\Phi}_{n, m}\right. & \left.+B_{i j m n}\left[\mathcal{U}_{j, i} \bar{\Phi}_{n, m}+\overline{\mathcal{U}}_{j, i} \Phi_{n, m}\right]-\varrho \omega^{2} U_{i} \bar{U}_{i}-I_{i j} \omega^{2} \Phi_{i} \bar{\Phi}_{j}\right\} \\
& +\iota \omega D_{i j}\left(\bar{T} \mathcal{U}_{j, i}-T \overline{\mathcal{U}}_{j, i}\right)+\iota \omega E_{i j}\left(\bar{T} \Phi_{i, j}-T \bar{\Phi}_{i, j}\right) \\
= & \left\{\left[A_{i j m n} \mathcal{U}_{n, m}+B_{i j m n} \Phi_{n, m}-\iota \omega D_{i j} T\right] \bar{U}_{i}\right\}_{, j} \\
& +\left\{\left[A_{i j m n} \overline{\mathcal{U}}_{n, m}+B_{i j m n} \bar{\Phi}_{n, m}+\iota \omega D_{i j} \bar{T}\right] U_{i}\right\}_{, j} \\
& +\left\{\left[B_{m n i j} \mathcal{U}_{n, m}+C_{i j m n} \Phi_{n, m}-\iota \omega E_{i j} T\right] \bar{\Phi}_{i}\right\}_{, j} \\
& +\left\{\left[B_{m n i j} \overline{\mathcal{U}}_{n, m}+C_{i j m n} \bar{\Phi}_{n, m}+\iota \omega E_{i j} \bar{T}\right] \Phi_{i}\right\}_{, j} .
\end{aligned}
$$

Integrate equality (21) over $D\left(x_{3}\right)$, apply the divergence theorem, and use the lateral conditions (14); we get the equality (16).

Now, if we again consider equations $(13)_{1}$ and $(13)_{2}$ then it is easy to prove the following equality:

$$
\begin{aligned}
\{ & {\left.\left[A_{i j m n} \mathcal{U}_{n, m}+B_{i j m n} \Phi_{n, m}-\iota \omega D_{i j} T\right]_{, j}+\varrho \omega^{2} U_{i}\right\} \bar{U}_{i} } \\
& -\left\{\left[A_{i j m n} \overline{\mathcal{U}}_{n, m}+B_{i j m n} \bar{\Phi}_{n, m}+\iota \omega D_{i j} \bar{T}\right]_{, j}+\varrho \omega^{2} \bar{U}_{i}\right\} U_{i} \\
& +\left[B_{m n i j} \mathcal{U}_{n, m}+C_{i j m n} \Phi_{n, m}-\iota \omega E_{i j} T\right]_{, j} \bar{\Phi}_{i} \\
& +\varepsilon_{i j k}\left[A_{j k m n} \mathcal{U}_{n, m}+B_{j k m n} \Phi_{n, m}-\iota \omega D_{j k} T\right] \bar{\Phi}_{i}+I_{i j} \omega^{2} \Phi_{i} \bar{\Phi}_{j} \\
& -\left[B_{m n i j} \overline{\mathcal{U}}_{n, m}+C_{i j m n} \bar{\Phi}_{n, m}+\iota \omega E_{i j} \bar{T}\right]_{, j} \Phi_{i} \\
& -\varepsilon_{i j k}\left[A_{j k m n} \overline{\mathcal{U}}_{n, m}+B_{j k m n} \bar{\Phi}_{n, m}+\iota \omega D_{j k} \bar{T}\right] \Phi_{i}-I_{i j} \omega^{2} \Phi_{i} \bar{\Phi}_{j}=0 .
\end{aligned}
$$

With some calculations, equality (22) can be written in the form

$$
\begin{aligned}
\iota \omega D_{i j} & \left(\bar{T} \mathcal{U}_{j, i}+T \overline{\mathcal{U}}_{j, i}\right)+\iota \omega E_{i j}\left(\bar{T} \Phi_{i, j}+T \bar{\Phi}_{i, j}\right) \\
= & +\left\{\left[A_{i j m n} \overline{\mathcal{U}}_{n, m}+B_{i j m n} \bar{\Phi}_{n, m}+\iota \omega D_{i j} \bar{T}\right] U_{i}\right\}_{, j} \\
& -\left\{\left[A_{i j m n} \mathcal{U}_{n, m}+B_{i j m n} \Phi_{n, m}-\iota \omega D_{i j} T\right] \bar{U}_{i}\right\}_{, j} \\
& +\left\{\left[B_{m n i j} \overline{\mathcal{U}}_{n, m}+C_{i j m n} \bar{\Phi}_{n, m}+\iota \omega E_{i j} \bar{T}\right] \Phi_{i}\right\}_{, j} \\
& -\left\{\left[B_{m n i j} \mathcal{U}_{n, m}+C_{i j m n} \Phi_{n, m}-\iota \omega E_{i j} T\right] \bar{\Phi}_{i}\right\}_{, j} .
\end{aligned}
$$


Integrate equality (23) over $D\left(x_{3}\right)$, apply the divergence theorem; if we use the lateral conditions (14) we get the equality (17).

With the help of equation (13) ${ }_{3}$, we can deduce immediately the equality

$$
\begin{aligned}
& \bar{T}\left[\left(\frac{1}{\theta_{0}} K_{i j} T_{, j}\right)_{, i}-\iota \omega D_{i j} \mathcal{U}_{j, i}-\iota \omega E_{i j} \Phi_{j, i}+\frac{c}{\theta_{0}} \omega^{2} T\right] \\
& \quad+T\left[\left(\frac{1}{\theta_{0}} K_{i j} \bar{T}_{, j}\right)_{, i}+\iota \omega D_{i j} \mathcal{U}_{j, i}+\iota \omega E_{i j} \bar{\Phi}_{j, i}+\frac{c}{\theta_{0}} \omega^{2} \bar{T}\right]=0 .
\end{aligned}
$$

With some calculations, equality (24) can be written in the form

$$
\begin{aligned}
& \frac{2}{\theta_{0}}\left(K_{i j} T_{, i} \bar{T}_{, j}-c \omega^{2} T \bar{T}\right)+\iota \omega D_{i j}\left(\mathcal{U}_{j, i} \bar{T}-\mathcal{U}_{j, i} T\right)+\iota \omega E_{i j}\left(\Phi_{j, i} \bar{T}-\bar{\Phi}_{j, i} T\right) \\
& \quad=\left[\frac{1}{\theta_{0}} K_{i j}\left(\bar{T} T_{, j}+T \bar{T}_{, j}\right)\right]_{, i}
\end{aligned}
$$

Integrate equality (25) over $D\left(x_{3}\right)$, apply the divergence theorem, and if we use the lateral conditions (14) we get the equality (18).

Finally, we use again equation $(13)_{3}$ thus we will obtain, in a trivial way, the equality

$$
\begin{aligned}
\bar{T} & {\left[\left(\frac{1}{\theta_{0}} K_{i j} T_{, j}\right)_{, i}-\iota \omega D_{i j} \mathcal{U}_{j, i}-\iota \omega E_{i j} \Phi_{j, i}+\frac{c}{\theta_{0}} \omega^{2} T\right] } \\
& -T\left[\left(\frac{1}{\theta_{0}} K_{i j} \bar{T}_{, j}\right)_{, i}+\iota \omega D_{i j} \mathcal{U}_{j, i}+\iota \omega E_{i j} \bar{\Phi}_{j, i}+\frac{c}{\theta_{0}} \omega^{2} \bar{T}\right]=0 .
\end{aligned}
$$

With some calculations, equality (26) can be written in the form

$$
\iota \omega D_{i j}\left(\mathcal{U}_{j, i} \bar{T}+\mathcal{U}_{j, i} T\right)+\iota \omega E_{i j}\left(\bar{\Phi}_{j, i} T+\Phi_{j, i} \bar{T}\right)=\left[\frac{1}{\theta_{0}} K_{i j}\left(\bar{T} T_{, j}-T \bar{T}_{, j}\right)\right]_{, i} .
$$

Integrate equality (27) over $D\left(x_{3}\right)$, apply the divergence theorem; if we use the lateral conditions (14) we get the equality (19) and the proof of Theorem 1 is completed.

The next theorem is also dedicated to a proof of two auxiliary identities on which will be based the main result.

Theorem 2 Let $\left(U_{i}, \Phi_{i}, T\right)$ be a solution of the boundary value problem consisting of equations (13)-(15). Then we have the identities

$$
\begin{aligned}
\int_{D\left(x_{3}\right)} & {\left[A_{i j m n} \mathcal{U}_{n, m} \overline{\mathcal{U}}_{j, i}+C_{i j m n} \Phi_{i, j} \bar{\Phi}_{n, m}\right] d A } \\
& +\int_{D\left(x_{3}\right)}\left\{B_{i j m n}\left[\mathcal{U}_{n, m} \bar{\Phi}_{i, j}+\overline{\mathcal{U}}_{n, m} \Phi_{i, j}\right]-3 \omega^{2}\left(\varrho U_{i} \bar{U}_{i}+I_{i j} \Phi_{i} \bar{\Phi}_{j}\right)\right\} d A \\
& -2 \iota \omega \int_{D\left(x_{3}\right)}\left\{D_{i j}\left(T \overline{\mathcal{U}}_{j, i}-\bar{T} \mathcal{U}_{j, i}\right)+E_{i j}\left(T \bar{\Phi}_{j, i}-\bar{T} \Phi_{j, i}\right)\right\} d A \\
& -\iota \omega \int_{D\left(x_{3}\right)}\left\{D_{i j} x_{p}\left(T_{, p} \overline{\mathcal{U}}_{j, i}-\bar{T}_{, p} \mathcal{U}_{j, i}\right)+E_{i j} x_{p}\left(T_{, p} \bar{\Phi}_{j, i}-\bar{T}_{, p} \Phi_{j, i}\right)\right\} d A
\end{aligned}
$$




$$
\begin{aligned}
& =-\frac{d}{d x_{3}} \int_{D\left(x_{3}\right)}\left\{\left[A_{3 j m n} \mathcal{U}_{n, m}+B_{3 j m n} \Phi_{n, m}-\iota \omega D_{3 j} T\right] x_{p} \bar{U}_{j, p}\right\} d A \\
& -\frac{d}{d x_{3}} \int_{D\left(x_{3}\right)}\left\{\left[A_{3 j m n} \overline{\mathcal{U}}_{n, m}+B_{3 j m n} \bar{\Phi}_{n, m}+\iota \omega D_{3 j} \bar{T}\right] x_{p} U_{j, p}\right\} d A \\
& -\frac{d}{d x_{3}} \int_{D\left(x_{3}\right)}\left\{\left[B_{3 j m n} \mathcal{U}_{n, m}+C_{3 j m n} \Phi_{n, m}-\iota \omega E_{3 j} T\right] x_{p} \bar{\Phi}_{j, p}\right\} d A \\
& -\frac{d}{d x_{3}} \int_{D\left(x_{3}\right)}\left\{\left[B_{3 j m n} \overline{\mathcal{U}}_{n, m}+C_{3 j m n} \bar{\Phi}_{n, m}+\iota \omega E_{3 j} \bar{T}\right] x_{p} \Phi_{j, p}\right\} d A \\
& +\frac{d}{d x_{3}} \int_{D\left(x_{3}\right)} x_{3}\left[A_{i j m n} \overline{\mathcal{U}}_{n, m} \overline{\mathcal{U}}_{j, i}+C_{i j m n} \Phi_{i, j} \bar{\Phi}_{n, m}\right] d A \\
& +\frac{d}{d x_{3}} \int_{D\left(x_{3}\right)} x_{3}\left\{B_{i j m n}\left[\mathcal{U}_{n, m} \bar{\Phi}_{i, j}+\overline{\mathcal{U}}_{n, m} \Phi_{i, j}\right]-\varrho \omega^{2} U_{i} \bar{U}_{i}\right\} d A \\
& -\frac{d}{d x_{3}} \int_{D\left(x_{3}\right)}\left\{\iota \omega x_{3} D_{i j}\left(T \overline{\mathcal{U}}_{j, i}-\bar{T} \mathcal{U}_{j, i}\right)\right\} d A \\
& -\frac{d}{d x_{3}} \int_{D\left(x_{3}\right)}\left\{\iota \omega x_{3} E_{i j}\left(T \bar{\Phi}_{j, i}-\bar{T} \Phi_{j, i}\right)-x_{3} I_{i j} \omega^{2} \Phi_{i} \bar{\Phi}_{j}\right\} d A \\
& +\int_{\partial D\left(x_{3}\right)} x_{p} n_{p}\left(A_{i \alpha m \beta} n_{\alpha} n_{\beta} \frac{\partial U_{i}}{\partial n} \frac{\partial \bar{U}_{m}}{\partial n}+B_{i \alpha m \beta} n_{\alpha} n_{\beta} \frac{\partial U_{i}}{\partial n} \frac{\partial \bar{\Phi}_{m}}{\partial n}\right. \\
& \left.+C_{i \alpha m \beta} n_{\alpha} n_{\beta} \frac{\partial \Phi_{i}}{\partial n} \frac{\partial \bar{\Phi}_{m}}{\partial n}\right) d s \\
& \int_{D\left(x_{3}\right)} \frac{1}{\theta_{0}}\left(K_{i j} T_{, i} \bar{T}_{, j}-3 c \omega^{2} T \bar{T}\right) d A \\
& +\int_{D\left(x_{3}\right)} \iota \omega E_{i j}\left(\bar{\Phi}_{j, i} T_{, p}-\Phi_{j, i} \bar{T}_{, p}\right) d A \\
& +\int_{D\left(x_{3}\right)} \iota \omega D_{i j} x_{p}\left(\mathcal{U}_{j, i} T_{, p}-\mathcal{U}_{j, i} \bar{T}_{, p}\right) d A \\
& +\int_{\partial D\left(x_{3}\right)} \frac{1}{\theta_{0}} x_{p} n_{p} K_{\alpha \beta} n_{\alpha} n_{\beta} \frac{\partial T}{\partial n} \frac{\partial \bar{T}}{\partial n} d s \\
& =-\frac{d}{d x_{3}} \int_{D\left(x_{3}\right)} \frac{1}{\theta_{0}}\left[x_{\alpha} K_{3 \beta}\left(\bar{T}_{, \alpha} T_{, \beta}+T_{, \alpha} \bar{T}_{, \beta}\right)+x_{\alpha} K_{33}\left(T_{, 3} \bar{T}_{, \alpha}+\bar{T}_{3} T_{, \alpha}\right)\right] d A \\
& -\frac{d}{d x_{3}} \int_{D\left(x_{3}\right)} \frac{x_{3}}{\theta_{0}}\left(K_{33} T_{, 3} \bar{T}_{3}-K_{\alpha \beta} T_{, \alpha} \bar{T}_{, \beta}+c \omega^{2} T \bar{T}\right) d A .
\end{aligned}
$$

Proof Considering equations $(13)_{1}$ and $(13)_{2}$ it is easy to prove the following equality:

$$
\begin{aligned}
& \left\{\left[A_{i j m n} \mathcal{U}_{n, m}+B_{i j m n} \Phi_{n, m}-\iota \omega D_{i j} T\right]_{, j}+\varrho \omega^{2} U_{i}\right\} x_{p} \bar{U}_{i, p} \\
& \quad+\left[B_{m n i j} \mathcal{U}_{n, m}+C_{i j m n} \Phi_{n, m}-\iota \omega E_{i j} T\right]_{, j} x_{p} \bar{\Phi}_{i, p} \\
& \quad+\varepsilon_{i j k}\left[A_{j k m n} \mathcal{U}_{n, m}+B_{j k m n} \Phi_{n, m}-\iota \omega D_{j k} T\right] x_{p} \bar{\Phi}_{i, p}+I_{i j} \omega^{2} x_{p} \bar{\Phi}_{i, p} \Phi_{j} \\
& \quad+\left\{\left[A_{i j m n} \overline{\mathcal{U}}_{n, m}+B_{i j m n} \bar{\Phi}_{n, m}+\iota \omega D_{i j} \bar{T}\right]_{, j}+\varrho \omega^{2} \bar{U}_{i}\right\} x_{p} U_{i, p} \\
& \quad+\left[B_{m n i j} \overline{\mathcal{U}}_{n, m}+C_{i j m n} \bar{\Phi}_{n, m}+\iota \omega E_{i j} \bar{T}\right]_{, j} x_{p} \Phi_{i, p} \\
& \quad+\varepsilon_{i j k}\left[A_{j k m n} \overline{\mathcal{U}}_{n, m}+B_{j k m n} \bar{\Phi}_{n, m}+\iota \omega D_{j k} \bar{T}\right] x_{p} \Phi_{i, p}+I_{i j} \omega^{2} x_{p} \Phi_{i, p} \bar{\Phi}_{j}=0 .
\end{aligned}
$$


With simple calculations, equality (30) can be written in the form

$$
\begin{aligned}
\{ & {\left.\left[A_{i j m n} \mathcal{U}_{n, m}+B_{i j m n} \Phi_{n, m}-\iota \omega D_{i j} T\right] x_{p} \bar{U}_{i, p}\right\}_{, j} } \\
& -\left[A_{i j m n} \mathcal{U}_{n, m}+B_{i j m n} \Phi_{n, m}-\iota \omega D_{i j} T\right] x_{p} \bar{U}_{i, p j}+\varrho \omega^{2} x_{p} U_{i} \bar{U}_{i, p} \\
& +\left\{\left[B_{m n i j} \mathcal{U}_{n, m}+C_{i j m n} \Phi_{n, m}-\iota \omega E_{i j} T\right] x_{p} \bar{\Phi}_{i, p}\right\}_{, j} \\
& -\left[B_{m n i j} \mathcal{U}_{n, m}+C_{i j m n} \Phi_{n, m}-\iota \omega E_{i j} T\right] x_{p} \bar{\Phi}_{i, p j} \\
& +\varepsilon_{i j k}\left[A_{j k m n} \mathcal{U}_{n, m}+B_{j k m n} \Phi_{n, m}-\iota \omega D_{j k} T\right] x_{p} \bar{\Phi}_{i, p}+I_{i j} \omega^{2} x_{p} \bar{\Phi}_{i, p} \Phi_{j} \\
& +\left\{\left[A_{i j m n} \overline{\mathcal{U}}_{n, m}+B_{i j m n} \bar{\Phi}_{n, m}+\iota \omega D_{i j} \bar{T}\right] x_{p} U_{i, p}\right\}_{, j} \\
& -\left[A_{i j m n} \overline{\mathcal{U}}_{n, m}+B_{i j m n} \bar{\Phi}_{n, m}+\iota \omega D_{i j} \bar{T}\right] x_{p} U_{i, p j}+\varrho \omega^{2} x_{p} \bar{U}_{i} U_{i, p} \\
& +\left\{\left[B_{m n i j} \overline{\mathcal{U}}_{n, m}+C_{i j m n} \bar{\Phi}_{n, m}+\iota \omega E_{i j} \bar{T}\right] x_{p} \Phi_{i, p}\right\}_{, j} \\
& -\left[B_{m n i j} \overline{\mathcal{U}}_{n, m}+C_{i j m n} \bar{\Phi}_{n, m}+\iota \omega E_{i j} \bar{T}\right] x_{p} \Phi_{i, p j} \\
& +\varepsilon_{i j k}\left[A_{j k m n} \overline{\mathcal{U}}_{n, m}+B_{j k m n} \bar{\Phi}_{n, m}+\iota \omega D_{j k} \bar{T}\right] x_{p} \Phi_{i, p}+I_{i j} \omega^{2} x_{p} \Phi_{i, p} \bar{\Phi}_{j}=0 .
\end{aligned}
$$

This equality leads to

$$
\begin{aligned}
A_{i j m n} & \mathcal{U}_{n, m} \overline{\mathcal{U}}_{j, i}+C_{i j m n} \Phi_{i, j} \bar{\Phi}_{n, m} \\
& +B_{i j m n}\left(\mathcal{U}_{n, m} \bar{\Phi}_{i, j}+\overline{\mathcal{U}}_{n, m} \Phi_{i, j}\right)-3 \omega^{2}\left(\varrho U_{i} \bar{U}_{i}+I_{i j} \Phi_{i} \bar{\Phi}_{j}\right) \\
& -2 \iota \omega D_{i j}\left(T \overline{\mathcal{U}}_{j, i}-\bar{T} \mathcal{U}_{j, i}\right)-2 \iota \omega E_{i j}\left(T \bar{\Phi}_{j, i}-\bar{T} \Phi_{j, i}\right) \\
& -\iota \omega D_{i j} x_{p}\left(T_{, p} \overline{\mathcal{U}}_{j, i}-\bar{T}_{, p} \mathcal{U}_{j, i}\right)-\iota \omega E_{i j} x_{p}\left(T_{, p} \bar{\Phi}_{j, i}-\bar{T}_{, p} \Phi_{j, i}\right) \\
= & -\left\{\left[A_{i j m n} \mathcal{U}_{n, m}+B_{i j m n} \Phi_{n, m}-\iota \omega D_{i j} T\right] x_{p} \bar{U}_{i, p}\right\}_{, j} \\
& -\left\{\left[A_{i j m n} \overline{\mathcal{U}}_{n, m}+B_{i j m n} \bar{\Phi}_{n, m}+\iota \omega D_{i j} \bar{T}\right] x_{p} U_{i, p}\right\}_{, j} \\
& -\left\{\left[B_{m n i j} \mathcal{U}_{n, m}+C_{i j m n} \Phi_{n, m}-\iota \omega E_{i j} T\right] x_{p} \bar{\Phi}_{i, p}\right\}_{, j} \\
& -\left\{\left[B_{m n i j} \overline{\mathcal{U}}_{n, m}+C_{i j m n} \bar{\Phi}_{n, m}+\iota \omega E_{i j} \bar{T}\right] x_{p} \Phi_{i, p}\right\}_{, j} \\
& +\left[x_{k} A_{i j m n} \mathcal{U}_{n, m} \overline{\mathcal{U}}_{j, i}+x_{p} C_{i j m n} \Phi_{i, j} \bar{\Phi}_{n, m}\right]_{, p} \\
& +\left\{x_{p} B_{i j m n}\left[\mathcal{U}_{n, m} \bar{\Phi}_{i, j}+\overline{\mathcal{U}}_{n, m} \Phi_{i, j}\right]-x_{p} \varrho \omega^{2} U_{i} \bar{U}_{i}\right\}_{, p} \\
& -\left\{\iota \omega x_{p} D_{i j}\left(T \overline{\mathcal{U}}_{j, i}-\bar{T} \mathcal{U}_{j, i}\right)\right\}_{, p} \\
& -\left\{\iota \omega x_{p} E_{i j}\left(T \bar{\Phi}_{j, i}-\bar{T} \Phi_{j, i}\right)-x_{p} I_{i j} \omega^{2} \Phi_{i} \bar{\Phi}_{j}\right\}_{, p} .
\end{aligned}
$$

We integrate equality (32) and use the lateral boundary condition (14); then we are led to the equality

$$
\begin{aligned}
\int_{D\left(x_{3}\right)} & {\left[A_{i j m n} \overline{\mathcal{U}}_{n, m} \overline{\mathcal{U}}_{j, i}+C_{i j m n} \Phi_{i, j} \bar{\Phi}_{n, m}\right] d A } \\
& +\int_{D\left(x_{3}\right)}\left\{B_{i j m n}\left[\mathcal{U}_{n, m} \bar{\Phi}_{i, j}+\overline{\mathcal{U}}_{n, m} \Phi_{i, j}\right]-3 \omega^{2}\left(\varrho \mathcal{U}_{i} \overline{\mathcal{U}}_{i}+I_{i j} \Phi_{i} \bar{\Phi}_{j}\right)\right\} d A \\
& -2 \iota \omega \int_{D\left(x_{3}\right)}\left\{D_{i j}\left(T \overline{\mathcal{U}}_{j, i}-\bar{T} \mathcal{U}_{j, i}\right)+E_{i j}\left(T \bar{\Phi}_{j, i}-\bar{T} \Phi_{j, i}\right)\right\} d A
\end{aligned}
$$




$$
\begin{aligned}
& -\iota \omega \int_{D\left(x_{3}\right)}\left\{D_{i j} x_{p}\left(T_{, p} \overline{\mathcal{U}}_{j, i}-\bar{T}_{, p} \mathcal{U}_{j, i}\right)+E_{i j} x_{p}\left(T_{, p} \bar{\Phi}_{j, i}-\bar{T}_{, p} \Phi_{j, i}\right)\right\} d A \\
= & -\frac{d}{d x_{3}} \int_{D\left(x_{3}\right)}\left\{\left[A_{3 j m n} \mathcal{U}_{n, m}+B_{3 j m n} \Phi_{n, m}-\iota \omega D_{3 j} T\right] x_{p} \bar{U}_{j, p}\right\} d A \\
& -\frac{d}{d x_{3}} \int_{D\left(x_{3}\right)}\left\{\left[A_{3 j m n} \overline{\mathcal{U}}_{n, m}+B_{3 j m n} \bar{\Phi}_{n, m}+\iota \omega D_{3 j} \bar{T}\right] x_{p} U_{j, p}\right\} d A \\
& -\frac{d}{d x_{3}} \int_{D\left(x_{3}\right)}\left\{\left[B_{3 j m n} \mathcal{U}_{n, m}+C_{3 j m n} \Phi_{n, m}-\iota \omega E_{3 j} T\right] x_{p} \bar{\Phi}_{j, p}\right\} d A \\
& -\frac{d}{d x_{3}} \int_{D\left(x_{3}\right)}\left\{\left[B_{3 j m n} \overline{\mathcal{U}}_{n, m}+C_{3 j m n} \bar{\Phi}_{n, m}+\iota \omega E_{3 j} \bar{T}\right] x_{p} \Phi_{j, p}\right\} d A \\
& +\frac{d}{d x_{3}} \int_{D\left(x_{3}\right)} x_{3}\left[A_{i j m n} \overline{\mathcal{U}}_{n, m} \overline{\mathcal{U}}_{j, i}+C_{i j m n} \Phi_{i, j} \bar{\Phi}_{n, m}\right] d A \\
& +\frac{d}{d x_{3}} \int_{D\left(x_{3}\right)} x_{3}\left\{B_{i j m n}\left[\mathcal{U}_{n, m} \bar{\Phi}_{i, j}+\overline{\mathcal{U}}_{n, m} \Phi_{i, j}\right]-\varrho \omega^{2} U_{i} \overline{\mathcal{U}}_{i}\right\} d A \\
& -\frac{d}{d x_{3}} \int_{D\left(x_{3}\right)}\left\{\iota \omega x_{3} D_{i j}\left(T \overline{\mathcal{U}}_{j, i}-\bar{T} \mathcal{U}_{j, i}\right)\right\} d A \\
& -\frac{d}{d x_{3}} \int_{D\left(x_{3}\right)}\left\{\iota \omega x_{3} E_{i j}\left(T \bar{\Phi}_{j, i}-\bar{T} \Phi_{j, i}\right)-x_{3} I_{i j j} \omega^{2} \Phi_{i} \bar{\Phi}_{j}\right\} d A \\
& -\int_{\partial D\left(x_{3}\right)}\left[x_{p} \overline{\mathcal{U}}_{s, p} A_{p s m n} \overline{\mathcal{U}}_{n, m}+x_{p} \mathcal{U}_{s, p} A_{p s m n} \overline{\mathcal{U}}_{s, p}\right] n_{p} d s \\
& +\int_{\partial D\left(x_{3}\right)} x_{p} n_{p}\left\{A_{i j m n} \overline{\mathcal{U}}_{n, m} \mathcal{U}_{j, i}+C_{i j m n} \Phi_{j, i} \bar{\Phi}_{n, m}\right. \\
& \left.+\int_{i j m n}\left[\mathcal{U}_{j, i} \bar{\Phi}_{n, m}+\overline{\mathcal{U}}_{j, i} \Phi_{n, m}\right]\right\} d s . \\
& -\int_{\partial D\left(x_{3}\right)}\left[x_{p} \bar{\Phi}_{s, p} \bar{\Phi}_{p s m n} \mathcal{U}_{n, m}+x_{p} \Phi_{s, p} B_{p s m n} \overline{\mathcal{U}}_{n, m}\right] n_{p} d s \\
& \left.\Phi_{n, m}+x_{p} \Phi_{s, p} C_{p s m n} \bar{\Phi}_{n, m}\right] n_{p} d s \\
& \\
& \\
&
\end{aligned}
$$

If we take into account the lateral boundary condition (14) we conclude that

$$
U_{i, 3}=0 \quad \text { on } \partial D\left(x_{3}\right)
$$

On the curve $\partial D$ we have

$$
U_{i, \alpha}=n_{\alpha} \frac{\partial U_{i}}{\partial n}+\tau_{\alpha} \frac{\partial U_{i}}{\partial \tau}
$$

where $\tau_{\alpha}$ are components of the unit vector tangent to $\partial D$ and $\partial / \partial \tau$ is the tangential derivative. According to the lateral boundary condition (14) we deduce $\partial U_{i} / \partial \tau=0$ on the curve $\partial D$ and hence we obtain

$$
U_{i, \alpha}=n_{\alpha} \frac{\partial U_{i}}{\partial n} \quad \text { on the curve } \partial D
$$

Using equations (34) and (35), the last integral in (33) becomes 


$$
\begin{aligned}
& \int_{\partial D\left(x_{3}\right)} x_{p} n_{p}\left(A_{i j m n} \mathcal{U}_{j, i} \overline{\mathcal{U}}_{n, m}+B_{i j m n} \mathcal{U}_{j, i} \bar{\Phi}_{n, m}+C_{i j m n} \Phi_{j, i} \bar{\Phi}_{n, m}\right) d s \\
& =\int_{\partial D\left(x_{3}\right)} x_{p} n_{p}\left(A_{i \alpha m \beta} n_{\alpha} n_{\beta} \frac{\partial \mathcal{U}_{i}}{\partial n} \frac{\partial \overline{\mathcal{U}}_{m}}{\partial n}+B_{i \alpha m \beta} n_{\alpha} n_{\beta} \frac{\partial \mathcal{U}_{i}}{\partial n} \frac{\partial \bar{\Phi}_{m}}{\partial n}\right. \\
& \left.\quad+C_{i \alpha m \beta} n_{\alpha} n_{\beta} \frac{\partial \Phi_{i}}{\partial n} \frac{\partial \bar{\Phi}_{m}}{\partial n}\right) d s .
\end{aligned}
$$

For the other integrals in (33) we obtain

$$
\begin{gathered}
\int_{\partial D\left(x_{3}\right)}\left[x_{p} \overline{\mathcal{U}}_{s, p} A_{p s m n} \mathcal{U}_{n, m}+x_{p} \mathcal{U}_{s, p} A_{p s m n} \overline{\mathcal{U}}_{n, m}\right] n_{p} d s \\
=2 \int_{\partial D\left(x_{3}\right)} x_{p} n_{p} A_{i \alpha m \beta} n_{\alpha} n_{\beta} \frac{\partial \mathcal{U}_{i}}{\partial n} \frac{\partial \overline{\mathcal{U}}_{m}}{\partial n} d s, \\
\int_{\partial D\left(x_{3}\right)}\left[x_{p} \overline{\mathcal{U}}_{s, p} B_{p s m n} \Phi_{n, m}+x_{p} \mathcal{U}_{s, p} C_{p s m n} \bar{\Phi}_{n, m}\right] n_{p} d s \\
=2 \int_{\partial D\left(x_{3}\right)} x_{p} n_{p} B_{i \alpha m \beta} n_{\alpha} n_{\beta} \frac{\partial \mathcal{U}_{i}}{\partial n} \frac{\partial \bar{\Phi}_{m}}{\partial n} d s, \\
\int_{\partial D\left(x_{3}\right)}\left[x_{p} \bar{\Phi}_{s, p} C_{p s m n} \Phi_{n, m}+x_{p} \Phi_{s, p} C_{p s m n} \bar{\Phi}_{n, m}\right] n_{p} d s \\
=2 \int_{\partial D\left(x_{3}\right)} x_{p} n_{p} C_{i \alpha m \beta} n_{\alpha} n_{\beta} \frac{\partial \Phi_{i}}{\partial n} \frac{\partial \bar{\Phi}_{m}}{\partial n} d s .
\end{gathered}
$$

If we substitute the results of equations (36) and (37) in the equality (33), we obtain the first relation of Theorem 2, namely equation (28).

To prove equation (29) we start from the following equality, which is evident:

$$
\begin{aligned}
& x_{p} \bar{T}_{p}\left[\left(\frac{1}{\theta_{0}} K_{i j} T_{, j}\right)_{, i}-\iota \omega D_{i j} \mathcal{U}_{j, i}-\iota \omega E_{i j} \Phi_{j, i}+\frac{c}{\theta_{0}} \omega^{2} T\right] \\
& \quad+x_{p} T_{p}\left[\left(\frac{1}{\theta_{0}} K_{i j} \bar{T}_{, j}\right)_{, i}+\iota \omega D_{i j} \mathcal{U}_{j, i}-\iota \omega E_{i j} \bar{\Phi}_{j, i}+\frac{c}{\theta_{0}} \omega^{2} \bar{T}\right]=0 .
\end{aligned}
$$

After some direct calculations, equality (38) acquires the form

$$
\begin{aligned}
\iota \omega D_{i j} x_{p}\left(\mathcal{U}_{j, i} T_{, p}-\mathcal{U}_{j, i} \bar{T}_{, p}\right)+\iota \omega E_{i j} x_{p}\left(\bar{\Phi}_{j, i} T_{, p}-\Phi_{j, i} \bar{T}_{, p}\right) \\
=-x_{p}\left(\frac{c}{\theta_{0}} \omega^{2} T \bar{T}\right)_{, p}+\frac{2}{\theta_{0}} K_{i j} T_{, i} \bar{T}_{, j} \\
\quad-\left[\frac{1}{\theta_{0}} x_{p} K_{i j}\left(\bar{T}_{, p} T_{, j}+T_{, p} \bar{T}_{, j}\right)\right]_{, i}+x_{p}\left(\frac{1}{\theta_{0}} K_{i j} T_{, i} \bar{T}_{, j}\right)_{, p} .
\end{aligned}
$$

This equality can be rewritten as follows:

$$
\begin{gathered}
\frac{1}{\theta_{0}} K_{i j} T_{, i} \bar{T}_{, j}-\frac{3 c}{\theta_{0}} \omega^{2} T \bar{T}+\iota \omega E_{i j}\left(\bar{\Phi}_{j, i} T_{, p}-\Phi_{j, i} \bar{T}_{, p}\right)+\iota \omega D_{i j} x_{p}\left(\mathcal{U}_{j, i} T_{, p}-\mathcal{U}_{j, i} \bar{T}_{, p}\right) \\
=-\left(\frac{c}{\theta_{0}} \omega^{2} T \bar{T}\right)_{, p}-\left[\frac{1}{\theta_{0}} x_{p} K_{i j}\left(\bar{T}_{, p} T_{, j}+T_{, p} \bar{T}_{, j}\right)\right]_{, i}+\left(\frac{x_{p}}{\theta_{0}} K_{i j} T_{, i} \bar{T}_{, j}\right)_{, p}
\end{gathered}
$$


Now we integrate the equality (4) on $D\left(x_{3}\right)$ and, after using the lateral boundary condition (14), we are led to

$$
\begin{aligned}
\int_{D\left(x_{3}\right)} & \frac{1}{\theta_{0}}\left(K_{i j} T_{, i} \bar{T}_{, j}-3 c \omega^{2} T \bar{T}\right) d A+\int_{D\left(x_{3}\right)} \iota \omega E_{i j}\left(\bar{\Phi}_{j, i} T_{, p}-\Phi_{j, i} \bar{T}_{, p}\right) d A \\
& +\int_{D\left(x_{3}\right)} \iota \omega D_{i j} x_{p}\left(\mathcal{U}_{j, i} T_{, p}-\mathcal{U}_{j, i} \bar{T}_{, p}\right) d A \\
= & -\frac{d}{d x_{3}} \int_{D\left(x_{3}\right)}\left[\frac{1}{\theta_{0}} x_{p} K_{3 j}\left(\bar{T}_{, p} T_{, j}+T_{, p} \bar{T}_{, j}\right)-\frac{x_{3}}{\theta_{0}} K_{i j} T_{, i} \bar{T}_{, j}+\frac{x_{3}}{\theta_{0}} c \omega^{2} T \bar{T}\right] d A \\
& +\int_{\partial D\left(x_{3}\right)} \frac{1}{\theta_{0}}\left[x_{p} n_{p} K_{i j} T_{, i} \bar{T}_{, j}-x_{p} K_{p j}\left(\bar{T}_{, p} T_{, j}+T_{, p} \bar{T}_{, j}\right) n_{p}\right] d s .
\end{aligned}
$$

As we have already shown in the proof of equality (28), the lateral boundary condition implies

$$
T_{, 3}=0, \quad T_{, \alpha}=n_{\alpha} \frac{\partial T}{\partial n},
$$

on the curve $\partial D\left(x_{3}\right)$.

With these arguments, the equality (41) implies equation (29), therefore the proof of Theorem 2 is completed.

The conservation laws which will be proved in the following theorem will be used to derive a priori estimates for a solution of our mixed problem.

Theorem 3 Let $\left(U_{i}, \Phi_{i}, T\right)$ be a solution of the boundary value problem consisting of equations (13)-(15). Then the following two conservation laws are satisfied:

$$
\begin{aligned}
& \frac{d}{d x_{3}} \int_{D\left(x_{3}\right)} \omega^{2}\left(\varrho U_{j} \bar{U}_{j}+I_{i j} \Phi_{i} \bar{\Phi}_{j}+\frac{c}{\theta_{0}} T \bar{T}\right) d A \\
& \quad+\frac{d}{d x_{3}} \int_{D\left(x_{3}\right)} \frac{c}{\theta_{0}}\left(K_{33} T_{, 3} \bar{T}_{, 3}-K_{\alpha \beta} T_{, \alpha} \bar{T}_{, \beta}\right) d A \\
& \quad+\frac{d}{d x_{3}} \int_{D\left(x_{3}\right)}\left[A_{i 3 m 3} \mathcal{U}_{i, 3} \overline{\mathcal{U}}_{m, 3}+B_{i 3 m 3}\left(\mathcal{U}_{i, 3} \bar{\Phi}_{m, 3}+\overline{\mathcal{U}}_{i, 3} \Phi_{m, 3}\right)+C_{i 3 m 3} \Phi_{i, 3} \bar{\Phi}_{m, 3}\right] d A \\
& \quad-\frac{d}{d x_{3}} \int_{D\left(x_{3}\right)}\left[A_{i \alpha m \beta} \mathcal{U}_{i, \alpha} \overline{\mathcal{U}}_{m, \beta}+B_{i \alpha m 3}\left(\mathcal{U}_{i, \alpha} \bar{\Phi}_{m, \beta}+\overline{\mathcal{U}}_{i, \alpha} \Phi_{m, \beta}\right)\right. \\
& \left.\quad+C_{i \alpha m \beta} \Phi_{i, \alpha} \bar{\Phi}_{m, \beta}\right] d A \\
& \quad+\frac{d}{d x_{3}} \int_{D\left(x_{3}\right)}\left[\iota \omega D_{i \alpha}\left(T \overline{\mathcal{U}}_{i, \alpha}-\bar{T} \mathcal{U}_{i, \alpha}\right)+\iota \omega E_{i \alpha}\left(T \bar{\Phi}_{i, \alpha}-\bar{T} \Phi_{i, \alpha}\right)\right] d A=0 \\
& \frac{d}{d x_{3}} \int_{D\left(x_{3}\right)}\left\{\left[A_{3 j m n} \overline{\mathcal{U}}_{n, m}+B_{3 j m n} \bar{\Phi}_{n, m}+\iota \omega D_{3 j} \bar{T}\right] U_{j}\right\} d A \\
& \quad-\frac{d}{d x_{3}} \int_{D\left(x_{3}\right)}\left\{\left[A_{3 j m n} \mathcal{U}_{n, m}+B_{3 j m n} \Phi_{n, m}-\iota \omega D_{3 j} T\right] \bar{U}_{j}\right\} d A \\
& \quad+\frac{d}{d x_{3}} \int_{D\left(x_{3}\right)}\left\{\left[B_{3 j m n} \overline{\mathcal{U}}_{n, m}+C_{3 j m n} \bar{\Phi}_{n, m}+\iota \omega E_{3 j} \bar{T}\right] \Phi_{j}\right\} d A
\end{aligned}
$$




$$
\begin{aligned}
& -\frac{d}{d x_{3}} \int_{D\left(x_{3}\right)}\left\{\left[B_{3 j m n} \mathcal{U}_{n, m}+C_{3 j m n} \Phi_{n, m}-\iota \omega E_{3 j} T\right] \bar{\Phi}_{j}\right\} d A \\
= & \frac{d}{d x_{3}} \int_{D\left(x_{3}\right)}\left[\frac{1}{\theta_{0}} K_{3 j}\left(\bar{T} T_{, j}-T \bar{T}_{, j}\right)\right] d A .
\end{aligned}
$$

Proof To prove equation (42) we start by using equations $(13)_{1}$ and $(13)_{2}$; with the help of these we obtain the following equality:

$$
\begin{aligned}
\{ & {\left.\left[A_{i j m n} \mathcal{U}_{n, m}+B_{i j m n} \Phi_{n, m}-\iota \omega D_{i j} T\right]_{, i}+\varrho \omega^{2} U_{j}\right\} \bar{U}_{j, 3} } \\
& +\left\{\left[B_{i j m n} \mathcal{U}_{n, m}+C_{i j m n} \Phi_{n, m}-\iota \omega E_{i j} T\right]_{, i}\right. \\
& \left.+\varepsilon_{j i k}\left[A_{i k m n} \mathcal{U}_{n, m}+B_{i k m n} \Phi_{n, m}-\iota \omega D_{i k} T\right]+I_{i j} \omega^{2} \Phi_{i}\right\} \bar{\Phi}_{j, 3} \\
& +\left\{\left[A_{i j m n} \overline{\mathcal{U}}_{n, m}+B_{i j m n} \bar{\Phi}_{n, m}+\iota \omega D_{i j} \bar{T}\right]_{, i}+\varrho \omega^{2} \bar{U}_{j}\right\} U_{j, 3} \\
& +\left\{\left[B_{i j m n} \overline{\mathcal{U}}_{n, m}+C_{i j m n} \bar{\Phi}_{n, m}+\iota \omega E_{i j} \bar{T}\right]_{, i}\right. \\
& \left.+\varepsilon_{j i k}\left[A_{i k m n} \overline{\mathcal{U}}_{n, m}+B_{i k m n} \bar{\Phi}_{n, m}+\iota \omega D_{i k} \bar{T}\right]+I_{i j} \omega^{2} \bar{\Phi}_{i}\right\} \Phi_{j, 3}=0 .
\end{aligned}
$$

Performing direct calculations on equality (44) we are led to

$$
\begin{aligned}
& \frac{d}{d x_{3}}\left[\varrho \omega^{2} U_{j} \overline{\mathcal{U}}_{j}+I_{i j} \omega^{2} \Phi_{i} \bar{\Phi}_{j}+A_{i 3 m 3} \mathcal{U}_{i, 3} \overline{\mathcal{U}}_{m, 3}+B_{i 3 m 3}\left(\mathcal{U}_{i, 3} \bar{\Phi}_{m, 3}+\overline{\mathcal{U}}_{i, 3} \Phi_{m, 3}\right)\right. \\
& \quad+C_{i 3 m 3} \Phi_{i, 3} \bar{\Phi}_{m, 3}-A_{i \alpha m \beta} \mathcal{U}_{i, \alpha} \overline{\mathcal{U}}_{m, \beta}-B_{i \alpha m 3}\left(\mathcal{U}_{i, \alpha} \bar{\Phi}_{m, \beta}+\overline{\mathcal{U}}_{i, \alpha} \Phi_{m, \beta}\right) \\
& \left.\quad-C_{i \alpha m \beta} \Phi_{i, \alpha} \bar{\Phi}_{m, \beta}+\iota \omega D_{i \alpha}\left(T \overline{\mathcal{U}}_{i, \alpha}-\bar{T} \mathcal{U}_{i, \alpha}\right)+\iota \omega E_{i \alpha}\left(T \bar{\Phi}_{i, \alpha}-\bar{T} \Phi_{i, \alpha}\right)\right] \\
& \quad+\left[A_{i \alpha m 3} \mathcal{U}_{m, 3} \overline{\mathcal{U}}_{i, \alpha}+B_{i \alpha m 3}\left(\mathcal{U}_{m, 3} \bar{\Phi}_{i, \alpha}+\overline{\mathcal{U}}_{m, 3} \Phi_{i, \alpha}\right)+C_{i \alpha m 3} \Phi_{m, 3} \bar{\Phi}_{i, \alpha}\right]_{, \alpha} \\
& \quad+\left[\iota \omega D_{i \alpha}\left(\bar{T} \mathcal{U}_{i, 3}-T \overline{\mathcal{U}}_{i, 3}\right)\right]_{, \alpha}+\left[\iota \omega E_{i \alpha}\left(\bar{T} \Phi_{i, 3}-T \bar{\Phi}_{i, 3}\right)\right]_{, \alpha} \\
& \quad+\iota \omega D_{i j}\left(\bar{T}_{, 3} \mathcal{U}_{i, j}-T T_{, 3} \overline{\mathcal{U}}_{i, j}\right)+\iota \omega E_{i j}\left(\bar{T}_{, 3} \Phi_{i, j}-T_{, 3} \bar{\Phi}_{i, j}\right)=0 .
\end{aligned}
$$

Now integrate equality (45) and use the lateral boundary condition (14); we get

$$
\begin{aligned}
& \frac{d}{d x_{3}} \int_{D\left(x_{3}\right)}\left[\varrho \omega^{2} \mathcal{U}_{j} \overline{\mathcal{U}}_{j}+I_{i j} \omega^{2} \Phi_{i} \bar{\Phi}_{j}+A_{i 3 m 3} U_{i, 3} \overline{\mathcal{U}}_{m, 3}+B_{i 3 m 3}\left(\mathcal{U}_{i, 3} \bar{\Phi}_{m, 3}+\overline{\mathcal{U}}_{i, 3} \Phi_{m, 3}\right)\right. \\
& \quad+C_{i 3 m 3} \Phi_{i, 3} \bar{\Phi}_{m, 3}-A_{i \alpha m \beta} \mathcal{U}_{i, \alpha} \overline{\mathcal{U}}_{m, \beta}-B_{i \alpha m 3}\left(\mathcal{U}_{i, \alpha} \bar{\Phi}_{m, \beta}+\overline{\mathcal{U}}_{i, \alpha} \Phi_{m, \beta}\right) \\
& \left.\quad-C_{i \alpha m \beta} \Phi_{i, \alpha} \bar{\Phi}_{m, \beta}+\iota \omega D_{i \alpha}\left(T \overline{\mathcal{U}}_{i, \alpha}-\bar{T} \mathcal{U}_{i, \alpha}\right)+\iota \omega E_{i \alpha}\left(T \bar{\Phi}_{i, \alpha}-\bar{T} \Phi_{i, \alpha}\right)\right] d A \\
& \quad+\int_{D\left(x_{3}\right)}\left[\iota \omega D_{i j}\left(\bar{T}_{, 3} \mathcal{U}_{i, j}-T_{, 3} \overline{\mathcal{U}}_{i, j}\right)+\iota \omega E_{i j}\left(\bar{T}_{, 3} \Phi_{i, j}-T_{, 3} \bar{\Phi}_{i, j}\right)\right] d A=0 .
\end{aligned}
$$

Using equation $(13)_{3}$, it is clear that

$$
\begin{aligned}
& \bar{T}_{, 3} {\left[\frac{1}{\theta_{0}} K_{i j} T_{, i j}-\iota \omega\left(D_{i j} \mathcal{U}_{i, j}+E_{i j} \Phi_{i, j}\right)+\frac{c}{\theta_{0}} \omega^{2} T\right] } \\
&+T_{, 3}\left[\frac{1}{\theta_{0}} K_{i j} \bar{T}_{, i j}+\iota \omega\left(D_{i j} \overline{\mathcal{U}}_{i, j}+E_{i j} \bar{\Phi}_{i, j}\right)+\frac{c}{\theta_{0}} \omega^{2} \bar{T}\right]=0 .
\end{aligned}
$$


After doing some calculations, we can write equation (47) in the form

$$
\begin{aligned}
& \frac{d}{d x_{3}}\left(\frac{c}{\theta_{0}} \omega^{2} T \bar{T}+\frac{1}{\theta_{0}} K_{33} T_{, 3} \bar{T}_{, 3}-\frac{1}{\theta_{0}} K_{\alpha \beta} T_{, \alpha} \bar{T}_{, \beta}\right) \\
& \quad+\left(\frac{2}{\theta_{0}} K_{\alpha 3} T_{, 3} \bar{T}_{, 3}\right)_{, \alpha}+\iota \omega D_{i j}\left(T_{, 3} \overline{\mathcal{U}}_{i, j}-\bar{T}_{, 3} \mathcal{U}_{i, j}\right) \\
& \quad+\iota \omega E_{i j}\left(T_{, 3} \bar{\Phi}_{i, j}-\bar{T}_{, 3} \Phi_{i, j}\right)=0 .
\end{aligned}
$$

Now we integrate (48) on $D\left(x_{3}\right)$ and use the lateral boundary condition (14); we arrive at the equality

$$
\begin{aligned}
& \frac{d}{d x_{3}} \int_{D\left(x_{3}\right)}\left(\frac{c}{\theta_{0}} \omega^{2} T \bar{T}+\frac{1}{\theta_{0}} K_{33} T_{, 3} \bar{T}_{, 3}-\frac{1}{\theta_{0}} K_{\alpha \beta} T_{, \alpha} \bar{T}_{, \beta}\right) d A \\
& \quad+\int_{D\left(x_{3}\right)}\left[\iota \omega D_{i j}\left(T_{, 3} \overline{\mathcal{U}}_{i, j}-\bar{T}_{, 3} \mathcal{U}_{i, j}\right)+\iota \omega E_{i j}\left(T_{, 3} \bar{\Phi}_{i, j}-\bar{T}_{, 3} \Phi_{i, j}\right)\right] d A=0 .
\end{aligned}
$$

By using equations (49) and (46) we obtain the equality (42). The conservation law (43) is obtained immediately equaling the right-side members of equality (17) and (19). This concludes the proof of Theorem 3 .

Combining equalities (16)-(19) of Theorem 1 with equalities (28)-(29) of Theorem 2 and those of Theorem 3, namely (42)-(43), we obtain various measures associated with the amplitude $\left(U_{i}, \Phi_{i}, T\right)$. With the help of these measures, we will obtain suitable spatial estimates to describe the spatial behavior of the respective amplitude.

The next result is a first estimate which describes the spatial behavior of the solution.

Theorem 4 Let $\left(U_{i}, \Phi_{i}, T\right)$ be a solution of the boundary value problem consisting of equations (13)-(15). Then the following equality holds:

$$
\begin{aligned}
\int_{D\left(x_{3}\right)} & {\left[A_{i j m n} \mathcal{U}_{j, i} \overline{\mathcal{U}}_{n, m}+B_{i j m n}\left(\mathcal{U}_{j, i} \bar{\Phi}_{n, m}+\overline{\mathcal{U}}_{j, i} \Phi_{n, m}\right)+C_{i j m n} \Phi_{j, i} \bar{\Phi}_{n, m}\right.} \\
& \left.-\omega^{2}\left(\varrho \mathcal{U}_{i} \overline{\mathcal{U}}_{i}+I_{i j} \Phi_{i} \bar{\Phi}_{j}+\frac{c}{\theta_{0}} T \bar{T}\right)+\frac{c}{\theta_{0}} K_{i j} T_{, i} \bar{T}_{, j}\right] d A \\
& +\int_{D\left(x_{3}\right)}\left[\iota \omega D_{i j}\left(\bar{T} \mathcal{U}_{j, i}-T \overline{\mathcal{U}}_{j, i}\right)+\iota \omega E_{i j}\left(\bar{T} \Phi_{j, i}-T \bar{\Phi}_{j, i}\right)\right] d A \\
= & \frac{d}{d x_{3}} \int_{D\left(x_{3}\right)}\left\{\left[A_{3 j m n} \mathcal{U}_{n, m}+B_{3 j m n} \Phi_{n, m}-\iota \omega D_{3 j} T\right] \bar{U}_{j}\right\} d A \\
& +\frac{d}{d x_{3}} \int_{D\left(x_{3}\right)}\left\{\left[A_{3 j m n} \overline{\mathcal{U}}_{n, m}+B_{3 j m n} \bar{\Phi}_{n, m}+\iota \omega D_{3 j} \bar{T}\right] U_{j}\right\} d A \\
& +\frac{d}{d x_{3}} \int_{D\left(x_{3}\right)}\left\{\left[B_{3 j m n} \mathcal{U}_{n, m}+C_{3 j m n} \Phi_{n, m}-\iota \omega E_{3 j} T\right] \bar{\Phi}_{j}\right\} d A \\
& +\frac{d}{d x_{3}} \int_{D\left(x_{3}\right)}\left\{\left[B_{3 j m n} \overline{\mathcal{U}}_{n, m}+C_{3 j m n} \bar{\Phi}_{n, m}+\iota \omega E_{3 j} \bar{T}\right] \Phi_{j}\right\} d A \\
& +\frac{d}{d x_{3}} \int_{D\left(x_{3}\right)} \frac{1}{\theta_{0}} K_{33}\left(T \bar{T}_{, 3}+\bar{T}_{, 3}\right) d A .
\end{aligned}
$$


Proof By combining equations (16) and (18) we obtain immediately the above desired identity (50).

Another a priori estimate will be proved in the next theorem.

Theorem 5 If $\left(U_{i}, \Phi_{i}, T\right)$ is a solution of the boundary value problem consisting of equations (13)-(15), then we have

$$
\begin{aligned}
\int_{D\left(x_{3}\right)} & {\left[A_{i j m n} \mathcal{U}_{j, i} \overline{\mathcal{U}}_{n, m}+B_{i j m n}\left(\mathcal{U}_{j, i} \bar{\Phi}_{n, m}+\bar{U}_{j, i} \Phi_{n, m}\right)+C_{i j m n} \Phi_{j, i} \bar{\Phi}_{n, m}\right.} \\
& \left.+\frac{1}{\theta_{0}} K_{i j} T_{, i} \bar{T}_{, j}+\omega^{2}\left(\varrho U_{i} \bar{U}_{i}+I_{i j} \Phi_{i} \bar{\Phi}_{j}+\frac{1}{\theta_{0}} T \bar{T}\right)\right] d A \\
& -\int_{\partial D\left(x_{3}\right)} x_{p} n_{p}\left(A_{i \alpha m \beta} n_{\alpha} n_{\beta} \frac{\partial \mathcal{U}_{i}}{\partial n} \frac{\partial \overline{\mathcal{U}}_{m}}{\partial n}+B_{i \alpha m \beta} n_{\alpha} n_{\beta} \frac{\partial \mathcal{U}_{i}}{\partial n} \frac{\partial \bar{\Phi}_{m}}{\partial n}\right. \\
& \left.+C_{i \alpha m \beta} n_{\alpha} n_{\beta} \frac{\partial \Phi_{i}}{\partial n} \frac{\partial \bar{\Phi}_{m}}{\partial n}\right) d s-\int_{\partial D\left(x_{3}\right)} \frac{1}{\theta_{0}} x_{p} n_{p} K_{\alpha \beta} n_{\alpha} n_{\beta} \frac{\partial T}{\partial n} \frac{\partial \bar{T}}{\partial n} d s \\
= & \frac{d}{d x_{3}} \int_{D\left(x_{3}\right)}\left[\left(A_{3 j m n} \mathcal{U}_{n, m}+B_{3 j m n} \Phi_{n, m}-\iota \omega D_{3 j} T\right)\left(\overline{\mathcal{U}}_{j}+x_{p} \overline{\mathcal{U}}_{j, p}\right)\right. \\
& \left.+\left(A_{3 j m n} \overline{\mathcal{U}}_{n, m}+B_{3 j m n} \bar{\Phi}_{n, m}+\iota \omega D_{3 j} \bar{T}\right)\left(\mathcal{U}_{j}+x_{p} \mathcal{U}_{j, p}\right)\right] d A \\
& +\frac{d}{d x_{3}} \int_{D\left(x_{3}\right)}\left[\left(B_{3 j m n} \mathcal{U}_{n, m}+C_{3 j m n} \Phi_{n, m}-\iota \omega E_{3 j} T\right)\left(\bar{\Phi}_{j}+x_{p} \bar{\Phi}_{j, p}\right)\right. \\
& \left.+\left(B_{3 j m n} \overline{\mathcal{U}}_{n, m}+C_{3 j m n} \bar{\Phi}_{n, m}+\iota \omega E_{3 j} \bar{T}\right)\left(\Phi_{j}+x_{p} \Phi_{j, p}\right)\right] d A \\
& +\frac{d}{d x_{3}} \int_{D\left(x_{3}\right)} \frac{1}{\theta_{0}} K_{33}\left(T \bar{T}_{, 3}+\bar{T} T_{, 3}\right) d A \\
& +\frac{d}{d x_{3}} \int_{D\left(x_{3}\right)} \frac{x_{\alpha}}{\theta_{0}}\left[K_{3 \alpha}\left(\bar{T}_{, \alpha} T_{, \beta}+T_{, \alpha} \bar{T}_{, \beta}\right)+K_{33}\left(\bar{T}_{, \alpha} T_{, 3}+T_{, \alpha} \bar{T}_{, 3}\right)\right] d A \\
& +\frac{d}{d x_{3}} \int_{D\left(x_{3}\right)}\left\{x_{3}\left[A_{i 3 m 3} \mathcal{U}_{i, 3} \overline{\mathcal{U}}_{m, 3}+B_{i 3 m 3}\left(\mathcal{U}_{i, 3} \bar{\Phi}_{m, 3}+\overline{\mathcal{U}}_{i, 3} \Phi_{m, 3}\right)+C_{i 3 m 3} \Phi_{i, 3} \bar{\Phi}_{m, 3}\right]\right. \\
& +x_{3}\left[A_{i \alpha m \beta} \mathcal{U}_{i, \alpha} \overline{\mathcal{U}}_{m, \beta}+B_{i \alpha m \beta}\left(\mathcal{U}_{i, \alpha} \bar{\Phi}_{m, \beta}+\overline{\mathcal{U}}_{i, \alpha} \Phi_{m, \beta}\right)+C_{i \alpha m \beta} \Phi_{i, \alpha} \bar{\Phi}_{m, \beta}\right] \\
& +x_{3} \iota \omega\left[D_{i \alpha}\left(T \overline{\mathcal{U}}_{i, \alpha}-\bar{T} \mathcal{U}_{i, \alpha}\right)+E_{i \alpha}\left(T \bar{\Phi}_{i, \alpha}-\bar{T}_{\theta_{0}} \Phi_{i, \alpha}\right)\right] \\
& \left.\left.x_{33} T_{, 3} \bar{T}_{, 3}-K_{\alpha \beta} T_{, \alpha} \bar{T}_{, \beta}\right)+x_{3} \omega^{2}\left(\varrho U_{i} \bar{U}_{i}+I_{i j} \Phi_{i} \bar{\Phi}_{j}+\frac{c}{\theta_{0}} T \bar{T}\right)\right\} d A . \\
&
\end{aligned}
$$

Proof We arrive at the equality (51) if we combine the results from equalities (28) and (29) of Theorem 2 with equation (50) of Theorem 4.

The result of the spatial behavior will be based on equality (51). For the result to be rigorous, we specify assumptions which are really common in continuum mechanics. Thus, we assume that the tensors of the micropolar thermoelasticity satisfy the strong ellipticity condition,

$$
\begin{aligned}
& A_{i j m n} x_{i} x_{m} y_{j} y_{n}>0, \\
& B_{i j m n} x_{i} x_{m} y_{j} y_{n}>0, \quad \text { for all non-zero vectors }\left(x_{1}, x_{2}, x_{3}\right),\left(y_{1}, y_{2}, y_{3}\right), \\
& C_{i j m n} x_{i} x_{m} y_{j} y_{n}>0 .
\end{aligned}
$$


Also, the specific heat $c$ and the conductivity tensor $K_{i j}$ satisfy the conditions

$$
c>0, \quad K_{i j} x_{i} x_{j}>0, \quad \text { for all non-zero vector }\left(x_{1}, x_{2}, x_{3}\right) .
$$

It is clear that from (52) that we can deduce

$$
\begin{aligned}
& A_{i 3 m 3} x_{i} x_{m}>0, \\
& B_{i 3 m 3} x_{i} x_{m}>0, \quad \text { for all non-zero vector }\left(x_{1}, x_{2}, x_{3}\right), \\
& C_{i 3 m 3} x_{i} x_{m}>0 .
\end{aligned}
$$

Since the curve $\partial D$ was presumed regular, we deduce that there is $h_{0}>0$ such that $x_{p} n_{p} \geq$ $h_{0}>0$. Then we have the inequalities

$$
\begin{aligned}
0 \leq & \int_{\partial D\left(x_{3}\right)} x_{p} n_{p}\left(A_{i \alpha m \beta} n_{\alpha} n_{\beta} \frac{\partial U_{i}}{\partial n} \frac{\partial \bar{U}_{m}}{\partial n}+2 B_{i \alpha m \beta} n_{\alpha} n_{\beta} \frac{\partial U_{i}}{\partial n} \frac{\partial \bar{\Phi}_{m}}{\partial n}\right. \\
& \left.+C_{i \alpha m \beta} n_{\alpha} n_{\beta} \frac{\partial \Phi_{i}}{\partial n} \frac{\partial \bar{\Phi}_{m}}{\partial n}\right) d s \\
\leq & M C \int_{\partial D\left(x_{3}\right)}\left(\frac{\partial U_{i}}{\partial n} \frac{\partial \bar{U}_{i}}{\partial n}+\frac{\partial \Phi_{i}}{\partial n} \frac{\partial \bar{\Phi}_{i}}{\partial n}\right) d s,
\end{aligned}
$$

where we have used the notations

$$
\begin{aligned}
& C=\left(A_{i \alpha m \beta} A_{i \alpha m \beta}+2 B_{i \alpha m \beta} B_{i \alpha m \beta}+C_{i \alpha m \beta} C_{i \alpha m \beta}\right)^{1 / 2}, \\
& M=\sup _{\left(x_{1}, x_{2}\right) \in \partial D} \sqrt{\left(x_{1}^{2}+x_{2}^{2}\right)} .
\end{aligned}
$$

Also, for the conductivity tensor $K_{i j}$ we have

$$
0 \leq \int_{\partial D\left(x_{3}\right)} \frac{1}{\theta_{0}} x_{p} n_{p} K_{\alpha \beta} n_{\alpha} n_{\beta} \frac{\partial T}{\partial n} \frac{\partial \bar{T}}{\partial n} d s \leq \frac{M K}{\theta_{0}} \int_{\partial D\left(x_{3}\right)} \frac{\partial T}{\partial n} \frac{\partial \bar{T}}{\partial n} d s,
$$

where $M$ is defined in (57) and

$$
K=\left(K_{\alpha \beta} K_{\alpha \beta}\right)^{1 / 2} .
$$

Now we introduce the quantities $m_{0}, m_{1}, \omega_{0}^{*}$, and $\omega_{1}^{*}$ by

$$
\begin{aligned}
& m_{0}=\max _{x_{3} \in[0, L]} \frac{\int_{\partial D\left(x_{3}\right)}\left(\frac{\partial U_{i}}{\partial n} \frac{\partial \bar{U}_{i}}{\partial n}+\frac{\partial \Phi_{i}}{\partial n} \frac{\partial \bar{\Phi}_{i}}{\partial n}\right) d s}{\int_{D\left(x_{3}\right)}\left(U_{i} \bar{U}_{i}+\Phi_{i} \bar{\Phi}_{i}\right) d s}, \quad \omega_{0}^{*}=\frac{1}{\varrho} M C m_{0}, \\
& m_{1}=\max _{x_{3} \in[0, L]} \frac{\int_{\partial D\left(x_{3}\right)} \frac{\partial T}{\partial n} \frac{\partial \bar{T}}{\partial n} d s}{\int_{D\left(x_{3}\right)} T \bar{T} d s}, \quad \omega_{0}^{*}=\frac{1}{c} M K m_{1} .
\end{aligned}
$$

We can assume that

$$
\begin{aligned}
& \omega>\omega^{*}=\max \left\{\omega_{0}^{*}, \omega_{1}^{*}\right\}, \\
& m_{0} \leq m_{0}^{*}, \quad m_{1} \leq m_{1}^{*},
\end{aligned}
$$


where

$$
\begin{aligned}
& m_{0}^{*}=\max \frac{\int_{\partial D\left(x_{3}\right)}\left(\frac{\partial U_{i}}{\partial n} \frac{\partial \bar{U}_{i}}{\partial n}+\frac{\partial \Phi_{i}}{\partial n} \frac{\partial \bar{\Phi}_{i}}{\partial n}\right) d s}{\int_{D\left(x_{3}\right)}\left(U_{i} \bar{U}_{i}+\Phi_{i} \bar{\Phi}_{i}\right) d s}, \\
& m_{1}^{*}=\max _{T \in H_{0}^{1}(D)} \frac{\int_{\partial D\left(x_{3}\right)} \frac{\partial T}{\partial n} \frac{\partial \bar{T}}{\partial n} d s}{\int_{D\left(x_{3}\right)} T \bar{T} d s} .
\end{aligned}
$$

Here the maximum from $m_{0}^{*}$ is calculated for $U_{i} \in H_{0}^{1}(D), \Phi_{i} \in H_{0}^{1}(D)$, where $H_{0}^{1}(D)$ is the usual Sobolev space. In this way we obtain an explicit critical value for the frequency of the vibration, namely

$$
\omega^{*}=\max \left\{\frac{1}{\varrho} M C m_{0}^{*}, \frac{1}{c} M K m_{1}^{*}\right\} .
$$

Combining the results from equations (51), (55), (58), and (62) we obtain the following estimate of the spatial behavior of the amplitude $\left(U_{i}, \Phi_{i}, T\right)$ :

$$
\begin{aligned}
& \frac{d}{d x_{3}} \int_{D\left(x_{3}\right)}\left[\left(A_{3 j m n} \mathcal{U}_{n, m}+B_{3 j m n} \Phi_{n, m}-\iota \omega D_{3 j} T\right)\left(\overline{\mathcal{U}}_{j}+x_{p} \overline{\mathcal{U}}_{j, p}\right)\right. \\
& \left.+\left(A_{3 j m n} \overline{\mathcal{U}}_{n, m}+B_{3 j m n} \bar{\Phi}_{n, m}+\iota \omega D_{3 j} \bar{T}\right)\left(\mathcal{U}_{j}+x_{p} \mathcal{U}_{j, p}\right)\right] d A \\
& +\frac{d}{d x_{3}} \int_{D\left(x_{3}\right)}\left[\left(B_{3 j m n} \mathcal{U}_{n, m}+C_{3 j m n} \Phi_{n, m}-\iota \omega E_{3 j} T\right)\left(\bar{\Phi}_{j}+x_{p} \bar{\Phi}_{j, p}\right)\right. \\
& \left.+\left(B_{3 j m n} \overline{\mathcal{U}}_{n, m}+C_{3 j m n} \bar{\Phi}_{n, m}+\iota \omega E_{3 j} \bar{T}\right)\left(\Phi_{j}+x_{p} \Phi_{j, p}\right)\right] d A \\
& +\frac{d}{d x_{3}} \int_{D\left(x_{3}\right)} \frac{x_{\alpha}}{\theta_{0}}\left[K_{3 \alpha}\left(\bar{T}_{, \alpha} T_{, \beta}+T_{, \alpha} \bar{T}_{, \beta}\right)+K_{33}\left(\bar{T}_{, \alpha} T_{, 3}+T_{, \alpha} \bar{T}_{, 3}\right)\right] d A \\
& +\frac{d}{d x_{3}} \int_{D\left(x_{3}\right)} \frac{1}{\theta_{0}}\left[K_{33}\left(T \bar{T}_{, 3}+\bar{T} T_{, 3}\right)+x_{3} \omega^{2}\left(\varrho U_{i} \bar{U}_{i}+I_{i j} \Phi_{i} \bar{\Phi}_{j}+\frac{1}{\theta_{0}} T \bar{T}\right)\right] d A \\
& +\frac{d}{d x_{3}} \int_{D\left(x_{3}\right)}\left\{x_{3}\left[A_{i 3 m 3} \mathcal{U}_{i, 3} \overline{\mathcal{U}}_{m, 3}+B_{i 3 m 3}\left(\mathcal{U}_{i, 3} \bar{\Phi}_{m, 3}+\overline{\mathcal{U}}_{i, 3} \Phi_{m, 3}\right)+C_{i 3 m 3} \Phi_{i, 3} \bar{\Phi}_{m, 3}\right]\right. \\
& -x_{3}\left[A_{i \alpha m \beta} \mathcal{U}_{i, \alpha} \overline{\mathcal{U}}_{m, \beta}+B_{i \alpha m \beta}\left(\mathcal{U}_{i, \alpha} \bar{\Phi}_{m, \beta}+\overline{\mathcal{U}}_{i, \alpha} \Phi_{m, \beta}\right)+C_{i \alpha m \beta} \Phi_{i, \alpha} \bar{\Phi}_{m, \beta}\right] \\
& -x_{3} \iota \omega\left[D_{i \alpha}\left(T \overline{\mathcal{U}}_{i, \alpha}-\bar{T} \mathcal{U}_{i, \alpha}\right)+E_{i \alpha}\left(T \bar{\Phi}_{i, \alpha}-\bar{T} \Phi_{i, \alpha}\right)\right] \\
& \left.+\frac{x_{3}}{\theta_{0}}\left(K_{33} T_{, 3} \bar{T}_{, 3}-K_{\alpha \beta} T_{, \alpha} \bar{T}_{, \beta}\right)+x_{3} \omega^{2}\left(\varrho U_{i} \bar{U}_{i}+I_{i j} \Phi_{i} \bar{\Phi}_{j}+\frac{c}{\theta_{0}} T \bar{T}\right)\right\} d A \\
& \geq \int_{D\left(x_{3}\right)}\left[A_{i j m n} \mathcal{U}_{j, i} \overline{\mathcal{U}}_{n, m}+B_{i j m n}\left(\mathcal{U}_{j, i} \bar{\Phi}_{n, m}+\overline{\mathcal{U}}_{j, i} \Phi_{n, m}\right)\right. \\
& \left.+C_{i j m n} \Phi_{j, i} \bar{\Phi}_{n, m}+\frac{1}{\theta_{0}} K_{i j} T_{, i} \bar{T}_{, j}\right] d A .
\end{aligned}
$$

With this the proof of Theorem 5 is complete.

Conclusion It is appropriate to note that the differential inequality (66) is different from those used for a deduction of the estimates of Saint-Venant type. 
To deduce these estimates we used only the strong ellipticity assumptions for the thermoelastic coefficients.

Therefore, these results can be applied to a large scale of materials.

\section{Competing interests}

The authors declare that they have no competing interests.

\section{Authors' contributions}

All authors contributed equally to the writing of this paper. All authors read and approved the final manuscript.

\section{Author details}

${ }^{1}$ Department of Mathematics and Computer Science, Transilvania University of Brasov, Brasov, 500118, Romania. ${ }^{2}$ Department of Mathematics and Computer Science, Cankaya University, Ankara, Turkey. ${ }^{3}$ Institute of Space Sciences, Magurele, Bucharest, Romania.

Received: 1 March 2016 Accepted: 1 June 2016 Published online: 07 June 2016

\section{References}

1. Chandrasekharaiah, DS: Hyperbolic thermoelasticity: a review of recent literature. Appl. Mech. Rev. 51, 705-729 (1998)

2. Green, AE, Naghdi, PM: Thermoelasticity without energy dissipation. J. Elast. 31, 189-208 (1993)

3. Green, AE, Naghdi, PM: On thermodynamics and the nature of the second law. Proc. R. Soc. Lond. A 357, 253-270 (1977)

4. Nappa, L: Spatial decay estimates for the evolution equations of thermoelasticity without energy dissipation J. Therm. Stresses 21, 581-592 (1998)

5. Chandrasekharaiah, DS: A note on the uniqueness of solution in the linear theory of thermoelasticity without energy dissipation. J. Elast. 43, 279-283 (1996)

6. Iesan, D: On the theory of thermoelasticity without energy dissipation. J. Therm. Stresses 21, 295-307 (1998)

7. Quintanilla, R: On existence in thermoelasticity without energy dissipation. J. Therm. Stresses 25, 195-202 (2002)

8. Abbas, IA, Zenkour, AM: The effect of magnetic field on thermal shock problem for a fiber-reinforced anisotropic half-space using Green-Naghdi's theory. J. Comput. Theor. Nanosci. 12(3), 438-442 (2015)

9. Abbas, IA: Generalized magneto-thermoelastic interaction in a fiber-reinforced anisotropic hollow cylinder. Int. J. Thermophys. 33(3), 567-579 (2012)

10. Zenkour, AM, Abbas, IA: Magneto-thermoelastic response of an infinite functionally graded cylinder using the finite element method. J. Vib. Control 20(12), 1907-1919 (2014)

11. Marin, M: A temporally evolutionary equation in elasticity of micropolar bodies with voids. Sci. Bull. 'Politeh.' Univ. Buchar., Ser. A, Appl. Math. Phys. 60(3-4), 67-78 (1998)

12. Marin, M: On the minimum principle for dipolar materials with stretch. Nonlinear Anal., Real World Appl. 10(3), 1572-1578 (2009)

13. Marin, M, Agarwal, RP, Mahmoud, SR: Non-simple material problems addressed by the Lagrange's identity. Bound. Value Probl. 2013, Article ID 135 (2013). doi:10.1186/1687-2770-2013-135

14. Marin, M: On existence and uniqueness in thermoelasticity of micropolar bodies. C. R. Math. Acad. Sci. Paris 321(12), 475-480 (1995)

15. Marin, M: An evolutionary equation in thermoelasticity of dipolar bodies. J. Math. Phys. 40(3), 1391-1399 (1999)

16. Marin, M: A partition of energy in thermoelasticity of microstretch bodies. Nonlinear Anal., Real World Appl. 11(4), 2436-2447 (2010)

17. Anwar Beg, O, Zueco, J, Takhar, HS, Beg, TA: Network numerical simulation of impulsively-started transient radiation-convection heat and mass transfer in a saturated Darcy-Forchheimer porous medium. Nonlinear Anal., Model. Control 13(3), 281-303 (2008)

18. Mahapatra, TR, Pal, D, Mondal, S: Influence of thermal radiation on non-Darcian natural convection in a square cavity filled with fluid saturated porous medium of uniform porosity. Nonlinear Anal., Model. Control 17(2), 223-237 (2012)

19. Chirita, S: Spatial decay estimates for solutions describing harmonic vibrations in a thermoelastic cylinder. J. Therm. Stresses 18, 421-436 (1995)

20. Flavin, JN, Knops, RJ: Some spatial decay estimates in continuum dynamics. J. Elast. 17, 249-264 (1987)

21. Ciarletta, M: A theory of micropolar thermoelasticity without energy dissipation. J. Therm. Stresses 22, 581-594 (1999) 